\title{
Conserved POU-binding site linked to SP1-binding site within FZD5 promoter: Transcriptional mechanisms of FZD5 in undifferentiated human ES cells, fetal liver/spleen, adult colon, pancreatic islet, and diffuse-type gastric cancer
}

\author{
YURIKO KATOH $^{1}$ and MASARU KATOH ${ }^{2}$ \\ ${ }^{1}$ M\&M Medical BioInformatics, Hongo 113-0033; ${ }^{2}$ Genetics and Cell Biology Section, \\ National Cancer Center Research Institute, Tokyo 104-0045, Japan
}

Received November 8, 2006; Accepted December 15, 2006

\begin{abstract}
Canonical WNT signals are transduced through Frizzled (FZD) family receptor and LRP5/LRP6 co-receptor to upregulate FGF20, JAG1, DKK1, WISP1, CCND1 and MYC genes for cell-fate determination, while non-canonical WNT signals are transduced through FZD family receptor and ROR2/ PTK7/RYK co-receptor to activate RHOA/RHOU/RAC/ CDC42, JNK, PKC, NLK and NFAT signaling cascades for the regulation of tissue polarity, cell movement, and adhesion. We previously reported molecular cloning and characterization of human FZD5, which showed six amino-acid substitutions with human Hfz5. FZD5, functioning as WNT5A receptor, is the key molecule in the fields of oncology, regenerative medicine, cardiology, rheumatology, diabetology, and gastroenterology. Here, comparative integromics analyses on FZD5 orthologs were performed by using bioinformatics (Techint) and human intelligence (Humint). Chimpanzee FZD5 and cow $F z d 5$ genes were identified within NW_104292.1 and AC166656.2 genome sequences, respectively. FZD5 orthologs were seven-transmembrane proteins with extracellular Frizzled domain, leucine zipper motif around the 5th transmembrane domain, and cytoplasmic DVL- and PDZ-binding motifs. Ser523 and Ser529 around the DVL-binding motif of FZD5 orthologs were putative aPKC phosphorylation sites. POU5F1 (OCT4)-binding site linked to SP1-binding site within the 5'-promoter region of human FZD5 gene was evolutionarily conserved among mammalian FZD5 orthologs. POU5F1 was more related to POU2F and POU3F subfamily members. POU5F1 was preferentially expressed in undifferentiated human embryonic stem (ES) cells, pancreatic islet, and diffusetype gastric cancer. POU2F1 (OCT1) was expressed in ES
\end{abstract}

Correspondence to: Dr Masaru Katoh, Genetics and Cell Biology Section, National Cancer Center Research Institute, 5-1-1 Tsukiji, Chuo-ku, Tokyo 104-0045, Japan

E-mail: mkatoh-kkr@umin.ac.jp

Key words: WNT, Frizzled, theoretical biology, POU-domain transcription factor, Krüppel-like transcription factor, stem cell signaling network, systems medicine cells, fetal liver/spleen, adult colon, $P O U 2 F 2$ in ES cells, fetal liver/spleen, and $P O U 2 F 3$ in diffuse-type gastric cancer. Multiple SP1/KLF family members, other than KLF2 or KLF4, were expressed in undifferentiated human ES cells. Together, these facts indicate that POU5F1 and POU2F subfamily members play a pivotal role for the FZD5 expression in undifferentiated human ES cells, fetal liver/spleen, adult colon, pancreatic islet, and diffuse-type gastric cancer.

\section{Introduction}

Canonical WNT signals are transduced through Frizzled (FZD) family receptor and LRP5/LRP6 co-receptor to upregulate FGF20, JAG1, DKK1, WISP1, CCND1 and MYC genes for cell-fate determination (1-13), while non-canonical WNT signals are transduced through FZD family receptor and ROR2/PTK7/RYK co-receptor to activate RHOA/RHOU/ RAC/CDC42, JNK, PKC, NLK and NFAT signaling cascades for the regulation of tissue polarity, cell movement, and adhesion (14-20). WNT signals are context-dependently transduced to canonical and non-canonical signaling cascades. Cross-talk of WNT signaling pathway and FGF, Notch, Hedgehog and BMP/Nodal/TGFß signaling pathways constitute the stem-cell signaling network, which is implicated in embryogenesis and adult tissues homeostasis (21-31). Dysregulation of the stem cell signaling networks results in the pathological conditions, such as cancer, rheumatoid arthritis, and metabolic syndrome.

We previously reported molecular cloning and characterization of human FZD5 (32), which showed six amino-acid substitutions with human Hfz5 (33). FZD5, functioning as WNT5A receptor, is the key molecule in the fields of oncology, regenerative medicine, cardiology, rheumatology, diabetology, and gastroenterology. Here, comparative integromics analyses on FZD5 orthologs are described. Chimpanzee FZD5 and cow $F z d 5$ genes were identified and characterized in this study. In addition, comparative genomics analyses revealed conserved POU- and SP1/KLF-binding sites within the 5'-promoter region of FZD5 orthologs. POU5F1 and POU2F subfamily members were found to play a pivotal role for the expression of FZD5 in undifferentiated human embryonic stem (ES) cells, and other cells or tissues. 


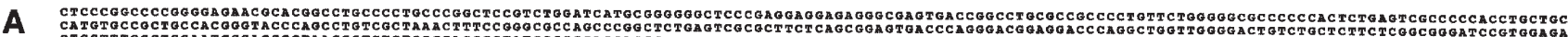

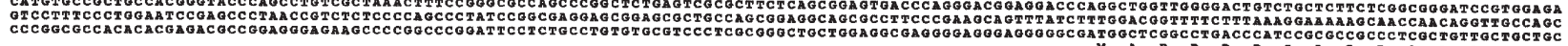

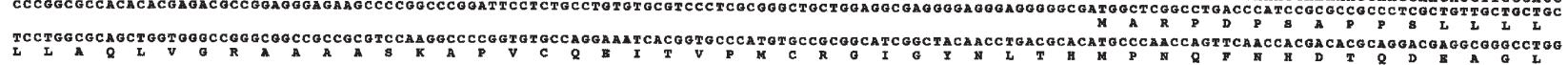

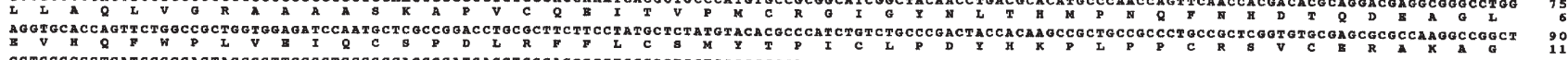

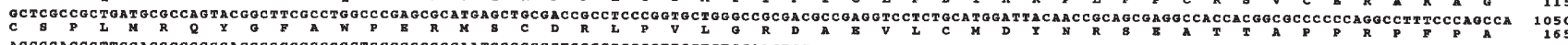

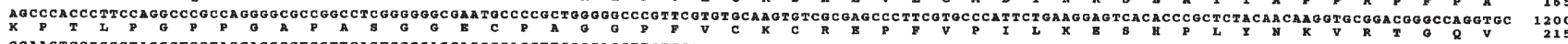

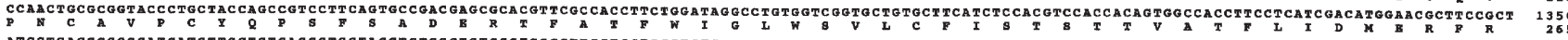

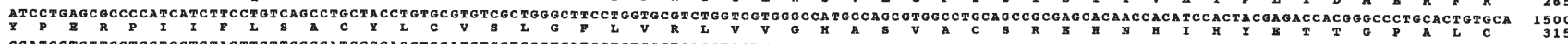

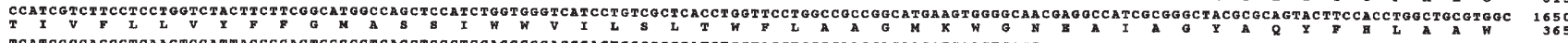

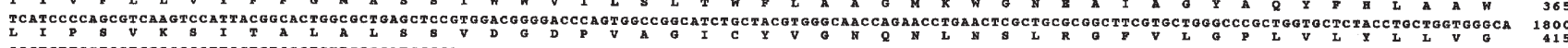

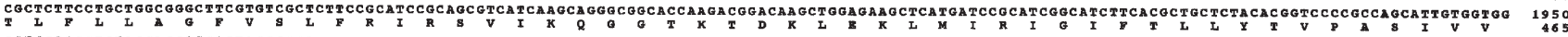

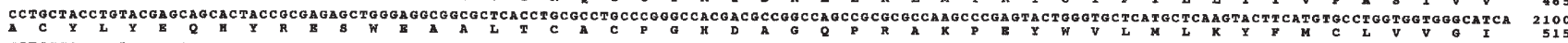

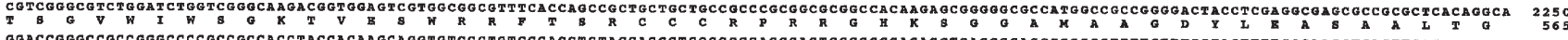

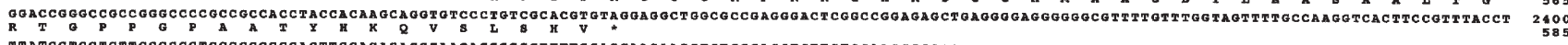

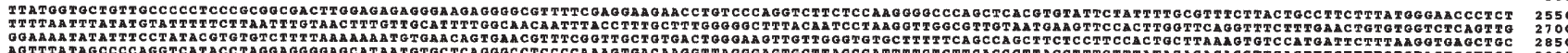

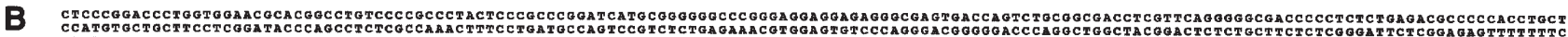

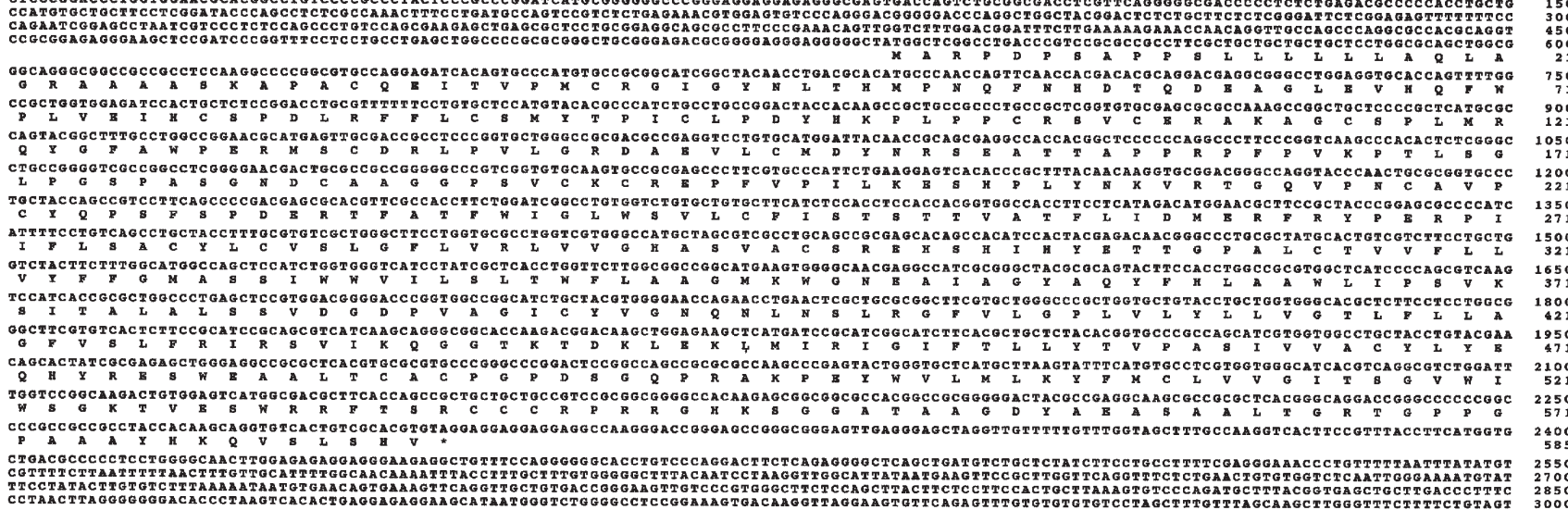

Figure 1. Chimpanzee FZD5 and cow Fzd5. Nucleotide position 1-3000 of complete coding sequences of chimpanzee FZD5 (A) and cow Fzd5 (B) are shown Nucleotides and amino-acid residues are numbered on the right.

\section{Materials and methods}

Identification and characterization of chimpanzee and cow FZD5 orthologs. Chimpanzee and cow genome sequences homologous to human FZD5 were searched for with BLAST programs as described previously (34-36). Exon-intron boundaries were determined based on the consensus sequence of exon-intron junctions ('gt ... ag' rule of intronic sequence) and codon usage within the coding region as described previously (37-39). Complete coding sequence (CDS) of chimpanzee FZD5 or cow Fzd5 was determined by assembling exonic regions.

Comparative genomics analyses. Human genome sequences around the FZD5 gene was compared with chimpanzee, cow, mouse and rat genome sequences to identify evolutionarily conserved regions. Binding sites for transcription factors, such as POU5F1, SOX2, NANOG and KLF, were then searched for.

Comparative proteomics analyses. Domain architecture of FZD5 orthologs were analyzed by using RPS-BLAST and PSORT II programs. Phylogenetic analyses on FZD and POU family proteins were performed by using the CLUSTALW program.

In silico expression analyses. Expressed sequence tags (ESTs) derived from human FZD5, POU family and SPI/ KLF family members were searched for by using the BLAST programs as described previously (40-42). The sources of human ESTs were listed up for in silico expression analyses.

\section{Results}

Chimpanzee FZD5 and cow Fzd5 genes. BLAST programs using human FZD5 RefSeq (NM_003468.3) revealed that chimpanzee FZD5 gene was located within NW_104292.1 genome sequence. Exon-intron boundaries of chimpanzee FZD5 gene were determined based on the consensus sequence of exon-intron junctions. Exon 1 corresponded to the nucleotide position 758775-758476 of NW_104292.1, while exons 2 to nucleotide position 758207-751775. Chimpanzee FZD5 gene was found consisting of two exons. Complete CDS of chimpanzee FZD5 was determined by assembling exonic regions. Genetyx program revealed that nucleotide position 555-2312 was the coding region. Chimpanzee FZD5 gene was found to encode a 585-amino-acid protein (Fig. 1A).

BLAST programs revealed that cow $F z d 5$ gene was located within AC166656.2 genome sequence. Complete CDS of cow Fzd5 was determined by assembling two exonic regions. Genetyx program revealed that nucleotide position 538-2295 was the coding region. Cow $F z d 5$ gene was found to encode a 585 -amino-acid protein (Fig. 1B).

Comparative proteomics analyses on FZD5 orthologs. Chimpanzee FZD5 and cow Fzd5 showed 99.7\% and 96.8\% total-amino-acid identity with human FZD5, respectively. Six 


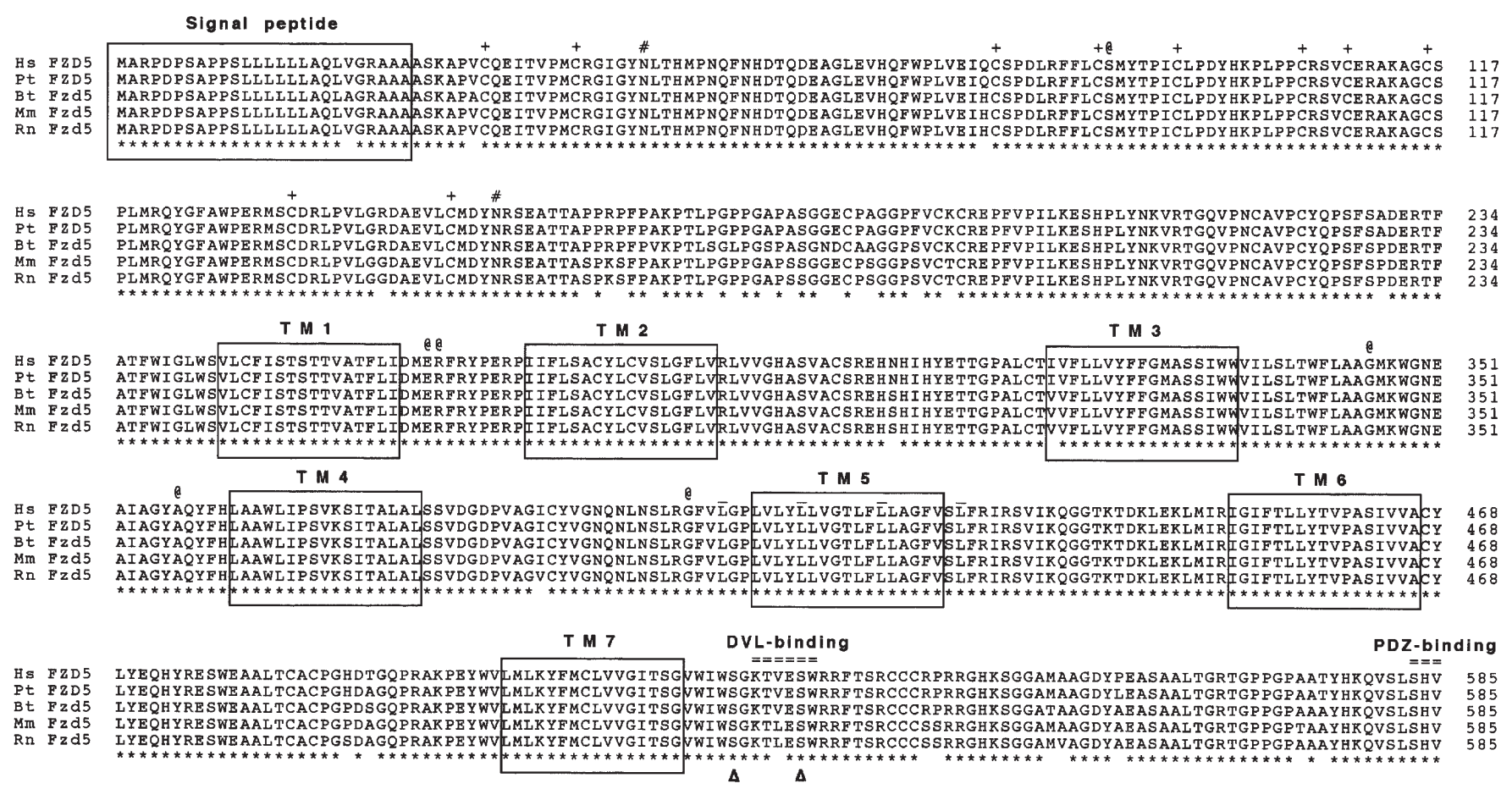

Figure 2. Mammalian FZD5 orthologs. Hs, human; Pt, chimpanzee; Bt, cow; Mm, mouse; Rn, rat. Signal peptide and seven-transmembrane domains (TM1-TM7) are boxed. Conserved Cys residues (cross) and Asn-linked glycosylation sites (sharp) within the N-terminal extracellular Frizzled region, leucine zipper motif around the TM5 domain (over line), DVL-binding and PZD-binding motifs within the C-terminal cytoplasmic region (double over line), Ser523 and Ser529 around the DVL-binding motif (open arrowhead) are conserved among mammalian FZD5 orthologs. Six amino-acid substitutions between human FZD5 and human Hzd5 (@) are also shown.

amino-acid substitutions occur between human FZD5 (32) and human Hfz5 (33). Because all of the six amino-acids corresponding to human FZD5 were conserved among mammalian FZD5 orthologs (Fig. 2), it was concluded that six aminoacid substitutions in $\mathrm{Hfz} 5$ are due to sequencing errors or cloning artifacts.

FZD5 orthologs were seven-transmembrane proteins with extracellular Frizzled domain, leucine zipper motif around the 5th transmembrane domain, and cytoplasmic DVL- and PDZ-binding motifs (Fig. 2). Asn47 and Asn151 within the $\mathrm{N}$-terminal extracellular region FZD5 orthologs were Asnlinked glycosylation sites. Ser523 and Ser529 around the DVLbinding motif of FZD5 orthologs were very similar to Ser554 and Ser560 of Drosophila Frizzled, which are phosphorylated by human aPKC (43).

In silico expression analysis on human FZD5. We have previously reported FZD5 mRNA expression in placenta, fetal liver, lung, kidney, adult pancreas, liver, colon, lung, kidney, K562 cells, SW480 cells, A549 cells, G361 cells, and HeLa S3 cells by using Northern blot analyses (32). We have also reported $F Z D 5$ mRNA expression in diffuse-type gastric cancer cells (44), and pancreatic islet (45). In this study, FZD5 mRNA was detected in pregnant uterus, placenta, amnion, undifferentiated human ES cells, fetal liver/spleen, adult colon, pancreatic islet, bone marrow stem cells, germ cell tumors, diffuse-type gastric cancer, colon cancer, and prostate cancer by using in silico expression analysis.

Transcriptional mechanism of FZD5 within undifferentiated human ES cells. Because FZD5 was preferentially expressed in undifferentiated human ES cells rather than differentiated human ES cells, POU5F1 (OCT4)-, SOX2-, and NANOGbinding sites around human FZD5 gene were searched for by using manual inspection. POU5F1-, SOX2- and NANOGbinding sites were identified within 5'-promoter region and 3'-UTR of human FZD5 gene. Among these sites, POU5F1binding site at about 600-bp upstream of transcriptional start site for human FZD5 gene was conserved in chimpanzee, cow, mouse, and rat FZD5 orthologs (Fig. 3A). NANOG-binding site at about 1500-bp upstream of transcriptional start site for human FZD5 gene was conserved in chimpanzee, cow and mouse $F Z D 5$ orthologs, but not in rat $F z d 5$ (Fig. 3A). Although SOX2-binding site was not linked to POU5F1-binding site within FZD5 5'-promoter region, SP1-binding site was linked to POU5F1-binding site with an interval of 3 bp (Fig. 3A). POU5F1-binding site linked to SP1-binding site within the 5'-promoter region of human FZD5 gene was evolutionarily conserved among mammalian $F Z D 5$ orthologs (Fig. 3A).

KLF4, KLF6, KLF7, KLF10, KLF12, KLF13, SP1, SP2, $S P 3$ and $S P 4$ promoters are bound by RNA polymerase II in undifferentiated human ES cells (46). In silico expression analyses revealed that $S P 1, S P 2, S P 3, K L F 10$, and $K L F 13$ were expressed in undifferentiated and differentiated human ES cells.

Together, these facts indicate that multiple SP1/KLF family members and POU5F1 are implicated in FZD5 expression in undifferentiated human ES cells, and also that POU5F1 plays a key role in the FZD5 expression in undifferentiated human ES cells.

Transcriptional mechanism of FZD5 by POU family members. Phylogenetic analyses on human POU family members revealed that POU5F1 was more similar to POU2F1, POU2F2, 

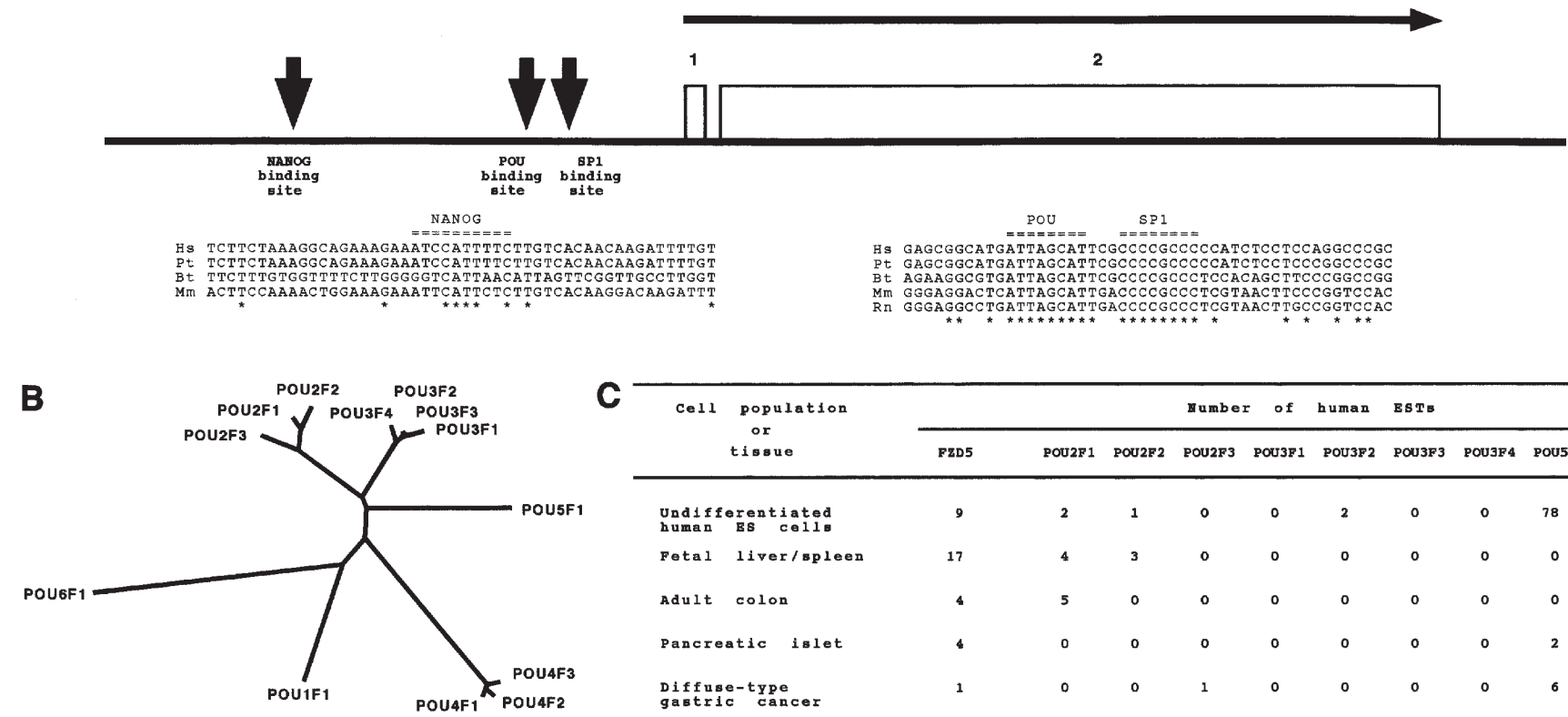

\begin{tabular}{|c|c|c|c|c|c|c|c|c|c|}
\hline \multirow{2}{*}{$\begin{array}{l}\text { population } \\
\text { or } \\
\text { tissue }\end{array}$} & \multicolumn{5}{|c|}{ Wumber } & human & \multicolumn{2}{|l|}{ ESTB } & \multirow[b]{2}{*}{ PousF } \\
\hline & FZD5 & POU $2 \mathrm{~F} 1$ & POU2F 2 & POU2F3 & POU3F 1 & POU3F 2 & POU3F 3 & POU3P4 & \\
\hline $\begin{array}{l}\text { Und } 1 \text { ferentiated } \\
\text { human ES celly }\end{array}$ & 9 & 2 & 1 & 0 & 0 & 2 & 0 & o & 78 \\
\hline Fetal liver/apleen & 17 & 4 & 3 & 0 & 0 & 0 & 0 & 0 & 0 \\
\hline Adult colon & 4 & 5 & 0 & 0 & 0 & 0 & 0 & 0 & 0 \\
\hline Pancreatic islet & 4 & 0 & 0 & 0 & 0 & 0 & $\circ$ & $\circ$ & 2 \\
\hline $\begin{array}{l}\text { Diffuse-type } \\
\text { gastric cancer }\end{array}$ & 1 & 0 & 0 & 1 & 0 & 0 & 0 & 0 & 6 \\
\hline
\end{tabular}

Figure 3. FZD5 transcription based on POU and KLF transcription factors. (A), Schematic structure of human FZD5 gene. POU- and KLF-binding sites with 3-bp spacing within the 5'-promoter is conserved among mammalian FZD5 orthologs. (B), Phylogenetic tree of human POU family transcription factors. POU5F1 is more related to POU2F and POU3F family members. (C), Expression profile of FZD5, and POU-domain transcription factors in human undifferentiated ES cells, fetal liver/spleen, adult colon, pancreatic islet, and diffuse-type gastric cancer.

POU2F3, POU3F1, POU3F2, POU3F3 and POU3F4 (Fig. 3B). POU5F1-binding sequence within the 5'-promoter of FZD5 gene was identical to that within the 3'-UTR of mouse $F g f 4$ gene, to which POU5F1, POU2F1 (OCT1) and POU3F1 (OCT6) can bind (47). Based on these facts, expression of POU5F1, POU2F1, POU2F2, POU2F3, POU3F1, POU3F2, $P O U 3 F 3$ and $P O U 3 F 4$ were next searched for to investigate the mechanism of FZD5 expression in fetal liver/spleen, adult colon, pancreatic islet, and diffuse-type gastric cancer. $P O U 5 F 1$ was preferentially expressed in pancreatic islet, and diffusetype gastric cancer. POU2F1 (OCT1) was expressed in fetal liver/spleen, and adult colon. POU2F2 was expressed in fetal liver/spleen. POU2F3 was expressed in diffuse-type gastric cancer. POU5F1 and POU2F subfamily members were implicated in FZD5 expression in fetal tissues, adult tissues, and cancer (Fig. 3C).

\section{Discussion}

Comparative integromics analyses on FZD5 orthologs were performed in this study, because FZD5 is the key molecule in a variety of fields, such as oncology, regenerative medicine, and gastroenterology. Chimpanzee FZD5 and cow $F z d 5$ genes were identified within NW_104292.1 and AC166656.2 genome sequences, respectively. Chimpanzee FZD5 and cow Fzd5 genes, consisting of two exons, encoded a 585-amino-acid protein (Fig. 1). Human FZD5 showed 99.7\% and 96.8\% total-amino-acid identity with chimpanzee FZD5 and cow Fzd5, respectively.

FZD5 orthologs were seven-transmembrane proteins with extracellular Frizzled domain, leucine zipper motif around the 5th transmembrane domain, and cytoplasmic DVL- and PDZ-binding motifs (Fig. 2). Leucine zipper motif around the 5th transmembrane domain might be implicated in homoor hetero-dimerization of Frizzled family members.

Ser523 and Ser529 around the DVL-binding motif within the C-terminal cytoplasmic region of FZD5 orthologs were putative aPKC phosphorylation sites. Because Ser554/Ser560 phosphorylation of Drosophila Frizzled by aPKC results in the inhibition of planar polarity signaling, Ser523 and Ser529 are critical amino-acid residues to determine the FZD5 signaling to canonical or non-canonical WNT signaling cascades.

POU5F1-binding site linked to SP1-binding site within the 5'-promoter region of human FZD5 gene was evolutionarily conserved among mammalian FZD5 orthologs (Fig. 3A). Klf4 and Klf2 are implicated in the maintenance of undifferentiated mouse ES cells (48), and Klf4 is also implicated in the ES-like transformation of mouse embryonic fibroblast (49). KLF1, KLF2 and KLF4, constituting a subfamily among KLF/SP1 family members, bind to CACCC motif rather than CGCCC motif (50). KLF1, KLF2 and KLF4 were not expressed in undifferentiated human ES cells, while $S P 1, S P 2, S P 3$, $K L F 10$, and $K L F 13$ were expressed in undifferentiated human ES cells as well as in differentiated human ES cells. Therefore, multiple SP1/KLF family members, other than KLF2 or KLF4, were implicated in FZD5 expression in undifferentiated human ES cells.

POU5F1 was preferentially expressed in undifferentiated human ES cells, pancreatic islet, and diffuse-type gastric cancer. $P O U 2 F 1$ was expressed in ES cells, fetal liver/spleen, adult colon, $P O U 2 F 2$ in ES cells, fetal liver/spleen, and $P O U 2 F 3$ in diffuse-type gastric cancer. Together, these facts indicate that POU5F1 and POU2F subfamily members play a pivotal role in the FZD5 expression in undifferentiated human ES cells, fetal liver/spleen, adult colon, pancreatic islet, and diffuse-type gastric cancer. 


\section{References}

1. Katoh M: WNT and FGF gene clusters. Int J Oncol 21: 1269-1273, 2002.

2. Katoh M and Katoh M: Cross-talk of WNT and FGF signaling pathways at GSK3ß to regulate $\beta$-catenin and SNAIL signaling cascades. Cancer Biol Ther 5: 1059-1064, 2006.

3. Pinson KI, Brennan J, Monkley S, et al: An LDL-receptor-related protein mediates Wnt signalling in mice. Nature 407: 535-538, 2000.

4. Kramps T, Peter O, Brunner E, et al: Wnt/wingless signaling requires $\mathrm{BCL}$ //legless-mediated recruitment of pygopus to the nuclear ß-catenin-TCF complex. Cell 109: 47-60, 2002.

5. Katoh $\mathrm{M}$ and Katoh $\mathrm{M}$ : Identification and characterization of human BCL9L gene and mouse $B c l 9 l$ gene in silico. Int $\mathrm{J}$ Mol Med 12: 643-649, 2003.

6. Chamorro MN, Schwartz DR, Vonica A, et al: FGF20 and DKK1 are transcriptional target of $\beta$-catenin and FGF20 is implicated in cancer and development. EMBO J 24: 73-84, 2005.

7. Katoh $\mathrm{M}$ and Katoh M: Comparative genomics on FGF20 orthologs. Oncol Rep 14: 287-290, 2005.

8. Katoh $\mathrm{M}$ and Katoh $\mathrm{M}$ : Notch ligand, JAG1, is evolutionarily conserved target of canonical WNT signaling pathway in progenitor cells. Int J Mol Med 17: 681-685, 2006.

9. Katoh $\mathrm{Y}$ and Katoh $\mathrm{M}$ : Comparative genomics on $D K K 1$ orthologs. Int J Oncol 27: 275-279, 2005.

10. Pennica D, Swanson TA, Welsh JW, et al: WISP genes are members of the connective tissue growth factor family that are up-regulated in Wnt1-transformed cells and aberrantly expressed in human colon tumors. Proc Natl Acad Sci USA 95: 14717-14722, 1998.

11. He TC, Sparks AB, Rago C, et al: Identification of $c-M Y C$ as a target of the APC pathway. Science 281: 1509-1512, 1998.

12. Tetsu $\mathrm{O}$ and McCormick F: $\beta$-catenin regulates expression of cyclin D1 in colon carcinoma cells. Nature 398: 422-426, 1999.

13. Swain RK, Katoh M, Medina A and Steinbeisser H: Xenopus frizzled-4S, a splicing variant of Xfz4, is a context-dependent activator and inhibitor of Wnt/ß-catenin signaling. Cell Commun Signal 3: 12, 2005.

14. Katoh M: WNT/PCP signaling pathway and human cancer. Oncol Rep 14: 1583-1588, 2005.

15. Yamaguchi TP, Bradley A, McMahon AP, et al: A Wnt5a pathway underlies outgrowth of multiple structures in the vertebrate embryo. Development 126: 1211-1223, 1999.

16. Oishi I, Suzuki H, Onishi N, et al: The receptor tyrosine kinase Ror2 is involved in non-canonical Wnt5a/JNK signalling pathway. Genes Cells 8: 645-654, 2003.

17. Lu X, Borchers AG, Jolicoeur C, et al: PTK7/CCK-4 is a novel regulator of planar cell polarity in vertebrates. Nature 430: 93-98, 2004.

18. Lu W, Yamamoto V, Ortega B, et al: Mammalian Ryk is a Wnt coreceptor required for stimulation of neurite outgrowth. Cell 119: 97-108, 2004

19. Boutros M, Paricio N, Strutt DI, et al: Dishevelled activates JNK and discriminates between JNK pathways in planar polarity and wingless signaling. Cell 94: 109-118, 1998.

20. Dejmek J, Safholm A, Kamp Nielsen C, et al: Wnt-5a/Ca ${ }^{2+}$ induced NFAT activity is counteracted by Wnt-5a/Yes-Cdc42casein kinase Ia signaling in human mammary epithelial cells. Mol Cell Biol 26: 6024-6036, 2006.

21. Clevers H: Stem cells, asymmetric division and cancer. Nat Genet 37: 1027-1028, 2005.

22. Katoh $\mathrm{M}$ and Katoh $\mathrm{M}$ : Comparative genomics on $F G F 8$, FGF17, and FGF18 orthologs. Int J Mol Med 16: 493-496, 2005.

23. Katoh Y and Katoh M: FGF signaling inhibitor, SPRY4, is evolutionarily conserved target of WNT signaling pathway in progenitor cells. Int J Mol Med 17: 529-532, 2006.

24. Katoh M and Katoh M: FGF signaling network in the gastrointestinal tract. Int J Oncol 29: 163-168, 2006.

25. Katoh M and Katoh M: NUMB is a break of WNT - Notch signaling cycle. Int J Mol Med 18: 517-521, 2006.
26. Garciadiego-Cazares D, Rosales C, Katoh M and ChimalMonroy J: Coordination of chondrocyte differentiation and joint formation by $\alpha 5 \beta 1$ integrin in the developing appendicular skeleton. Development 131: 4735-4742, 2004.

27. Katoh Y and Katoh M: Hedgehog signaling in gastric cancer. Cancer Biol Ther 4: 1050-1054, 2005.

28. Katoh Y and Katoh M: WNT antagonist, SFRP1, is Hedgehog signaling target. Int J Mol Med 17: 171-175, 2006.

29. Katoh Y and Katoh M: Comparative genomics on BMP4 orthologs. Int J Oncol 27: 581-585, 2005.

30. Katoh M and Katoh M: CER1 is a common target of WNT and NODAL signaling pathways in human embryonic stem cells. Int J Mol Med 17: 795-799, 2006.

31. Katoh Y and Katoh M: Comparative integromics on BMP/GDF family. Int J Mol Med 17: 951-955, 2006.

32. Saitoh T, Hirai M and Katoh M: Molecular cloning and characterization of human Frizzled-5 gene on chromosome 2q33.3q34 region. Int J Oncol 19: 105-110, 2001.

33. Wang Y, Macke JP, Abella BS, et al: A large family of putative transmembrane receptors homologous to the product of the Drosophila tissue polarity gene frizzled. J Biol Chem 271: 4468-4476, 1996.

34. Katoh M: Paradigm shift in gene-finding method: from benchtop approach to desk-top approach. Int J Mol Med 10: 677-682, 2002.

35. Katoh $M$ and Katoh M: Identification and characterization of human PRICKLE1 and PRICKLE2 genes as well as mouse Prickle1 and Prickle 2 genes homologous to Drosophila tissue polarity gene prickle. Int J Mol Med 11: 249-256, 2003.

36. Katoh $M$ and Katoh M: Identification and characterization of human DAPPER1 and DAPPER2 genes in silico. Int J Oncol 22: 907-913, 2003.

37. Katoh $\mathrm{M}$ and Katoh $\mathrm{M}$ : Identification and characterization of human FMNL1, FMNL2 and FMNL3 genes in silico. Int J Oncol 22: 1161-1168, 2003.

38. Katoh $\mathrm{M}$ and Katoh M: Identification and characterization of human HES2, HES3, and HES5 genes in silico. Int J Oncol 25: 529-534, 2004.

39. Katoh $\mathbf{M}$ and Katoh M: Identification and characterization of human HESL, rat Hesl and rainbow trout hesl genes in silico. Int J Mol Med 14: 747-751, 2005.

40. Katoh M: Molecular evolution of $W N T 2 B$ orthologs. Int J Oncol 26: 1135-1139, 2005.

41. Katoh $\mathrm{M}$ and Katoh $\mathrm{M}$ : Comparative genomics on FZD7 orthologs. Int J Mol Med 15: 1051-1055, 2005.

42. Katoh Y and Katoh M: Comparative genomics on HHIP family orthologs. Int J Mol Med 17: 391-395, 2006.

43. Djiane A, Yogev S and Mlodzik M: The apical determinants aPKC and dPatj regulate Frizzled-dependent planar cell polarity in the Drosophila eye. Cell 121: 621-631, 2005.

44. Kirikoshi H, Sekihara H and Katoh M: Expression profiles of 10 members of Frizzled gene family in human gastric cancer. Int J Oncol 19: 767-771, 2001.

45. Heller RS, Klein T, Ling Z, Heimberg H, Katoh M, Madsen OD and Serup P: Expression of WNT, Frizzled, $s F R P$, and $D K K$ genes in adult human pancreas. Gene Expr 11: 141-147, 2003.

46. Lee TI, Jenner RG, Boyer LA, et al: Control of developmental regulators by Polycomb in human embryonic stem cells. Cell 125: 301-313, 2006

47. Dailey L and Basilico C: Coevolution of HMG domains and Homeodomains and the generation of transcriptional regulation by Sox/POU complexes. J Cell Physiol 186: 315-328, 2001.

48. Nakatake Y, Fukui N, Iwamatsu Y, et al: Klf4 cooperates with Oct3/4 and Sox 2 to activate the Lefty1 core promoter in embryonic stem cells. Mol Cell Biol 26: 7772-7782, 2006.

49. Takahashi $\mathrm{K}$ and Yamanaka S: Induction of pluripotent stem cells from mouse embryonic and adult fibroblast cultures by defined factors. Cell 126: 663-676, 2006.

50. Kaczynski J, Cook T and Urrutia R: SpI- and Krüppel-like transcription factors. Genome Biol 4: 206, 2003. 